\title{
AN ASYMPTOTIC THEOREM FOR SYSTEMS OF LINEAR DIFFERENTIAL EQUATIONS
}

\author{
BY \\ ALLEN DEVINATZ $\left({ }^{1}\right)$
}

\begin{abstract}
Asymptotic estimates are obtained for a complete linearly independent set of solutions of a linear system of differential equations of the form

$$
y^{\prime}(t)=[A+V(t)+R(t)] y(t),
$$

where $A$ is a constant $n \times n$ matrix with $n$ distinct eigenvalues, $R(t)$ is an integrable matrix valued function on $(0, \infty)$ and $V(t)$ is an $n \times n$ matrix valued function having certain differentiability properties. The theorem that is obtained generalizes a theorem of N. Levinson, Duke Math. J. 15 (1948), 111-126.
\end{abstract}

1. In this paper we shall obtain asymptotic estimates for a complete linearly independent set of solutions of a linear system of differential equations of the form

$$
y^{\prime}(t)=[A+V(t)+R(t)] y(t),
$$

where $A$ is a constant $n \times n$ matrix with $n$ distinct eigenvalues, $R(t)$ is an integrable $n \times n$ matrix valued function on $(0, \infty)$ and $V(t)$ will be described in detail in the formal statement of the theorem.

A now classical asymptotic theorem of N. Levinson [5], [1, p. 92] gives asymptotic estimates for a linearly independent set of solutions of (1.1) when $V(t)$ is absolutely continuous on $(0, \infty)$, i.e., $V^{\prime}(t)$ is integrable on $(0, \infty), V(t) \rightarrow 0$ as $t \rightarrow \infty$ and certain conditions are fulfilled for the eigenvalues of $A+V(t)$. Our interest in this asymptotic theorem stems from its applicability to the problem of finding the deficiency index of an ordinary selfadjoint differential operator with real coefficients. In [2] (which is a corrected version of [3]) we showed how Levinson's theorem could be used to prove a general deficiency index theorem which contains as rather special cases all of the deficiency index theorems, obtained by asymptotic methods, which were extant in the literature up until 1966. In 1966 M. F. Fedorjuk [4] gave a deficiency index theorem, using asymptotic methods, which is not a consequence of Levinson's asymptotic theorem. However, Fedorjuk's theorem does not contain any of the other deficiency index theorems in the literature.

What we shall do here is obtain an asymptotic theorem which generalizes Levinson's asymptotic theorem and which can be used, by applying the methods of

Received by the editors September 9, 1970.

AMS 1969 subject classifications. Primary 3420.

Key words and phrases. Asymptotic estimates for solutions of systems of ordinary linear differential equations.

(1) Research partially supported by NSF Grant GP-9544.

Copyright (C) 1971, American Mathematical Society 
[2], to obtain a deficiency index theorem which very closely resembles Fedorjuk's deficiency index theorem. However, the exact connection between the two deficiency index theorems is not yet clear to us. We hope to be able to study this on another occasion.

We shall obtain our generalized asymptotic theorem by making use of an idea of Fedorjuk to reduce the problem to one in which Levinson's proof (but not his theorem) is applicable. Unfortunately, it is not possible to adapt Fedorjuk's proof to the more general situation.

2. Let us begin by stating the theorem which we shall prove.

THEOREM. Suppose $A$ is a constant $n \times n$ matrix which has $n$ distinct eigenvalues. Let $V(t)=V_{1}(t)+V_{2}(t)$ be $n \times n$ matrix valued functions defined on $(0, \infty)$ so that $V_{1}$ and $V_{2}^{\prime}$ are absolutely continuous on every compact subinterval of $(0, \infty), V_{1}(t)$, $V_{2}(t) \rightarrow 0$ as $t \rightarrow \infty$, and $\left|V_{1}^{\prime}(t)\right|,\left|V_{2}^{\prime}(t)\right|^{2},\left|V_{2}^{\prime \prime}(t)\right|$, and $\left|V_{2}^{\prime}(t)\right||V(t)|$ are integrable $\left({ }^{2}\right)$. Let $\left\{\lambda_{k}: 1 \leqq k \leqq n\right\}$ be the distinct eigenvalues of $A$ and $\left\{\lambda_{k}(t): 1 \leqq k \leqq n\right\}$ be the distinct eigenvalues of $A+V(t)$ (for all sufficiently large $t$ ) which have been chosen in such a manner that $\lambda_{k}(t) \rightarrow \lambda_{k}$ as $t \rightarrow \infty$. Further, let $R(t)$ be an integrable matrix valued function on $(0, \infty)$.

For a given integer $k, 1 \leqq k \leqq n$, let us set

$$
d_{k j}(t)=\operatorname{Re}\left[\lambda_{k}(t)-\lambda_{j}(t)\right],
$$

and suppose that all $j, 1 \leqq j \leqq n$, fall into one of two classes $I_{1 k}$ and $I_{2 k}$, where

$$
\begin{aligned}
& j \in I_{1 k} \quad \text { if } \int_{0}^{t} d_{k j}(\tau) d \tau \rightarrow \infty \text { as } t \rightarrow \infty \text { and } \int_{t_{1}}^{t_{2}} d_{k j}(\tau) d \tau \geqq-K\left(t_{2} \geqq t_{1} \geqq s_{0}\right), \\
& j \in I_{2 k} \quad \text { if } \int_{t_{1}}^{t_{2}} d_{k j}(\tau) d \tau<K\left(t_{2} \geqq t_{1} \geqq s_{0}\right),
\end{aligned}
$$

where $K$ is a constant and $s_{0}$ is some positive real number. Let $p_{k}$ be an eigenvector associated with $\lambda_{k}$. Then there is a solution $y_{k}$ of the differential equation (1.1) and a $t_{0} \geqq 0$, so that

$$
\lim _{t \rightarrow \infty} y_{k}(t) \exp \left\{-\int_{t_{0}}^{t} \lambda_{k}(\tau) d \tau\right\}=p_{k} .
$$

The proof of this theorem is an immediate consequence of the following proposition and a method used by Levinson in the proof of his asymptotic theorem. A comparison of the latter theorem with the theorem given in this paper shows that with the exception of the conditions on $V(t)$ all of the other hypotheses are the same.

Proposition. Suppose $A, V(t)$, and $R(t)$ satisfy the hypotheses of the previously stated theorem. Then there exists an invertible matrix valued function $P(t)$ so that

$\left.{ }^{2}\right)$ If $A$ is a matrix with entries $a_{j k}$, then we set $|A|=\sum_{j, k}\left|a_{j k}\right|$. A function is said to be integrable if it is integrable in some interval $(t, \infty), t \geqq 0$. 
$P(t) \rightarrow P$ as $t \rightarrow \infty$, where the columns of $P$ form a given linearly independent set of eigenvectors for $A, P(t)$ is absolutely continuous on every compact subinterval of $(0, \infty)$, and

$$
P(t)^{-1}[A+V(t)+R(t)] P(t)-P(t)^{-1} P^{\prime}(t)=\Lambda(t)+R_{1}(t),
$$

where $R_{1}(t)$ is integrable, and $\Lambda(t)$ is a diagonal matrix whose diagonal elements are $\lambda_{j}(t), 1 \leqq j \leqq n$.

Let us, for the moment, assume the proposition is true and give a proof of the theorem. The proof of the proposition will follow afterwards.

Proof of Theorem. Suppose $y$ is a solution of (1.1). Make the transformation $y(t)=P(t) x(t)$. We then get

$$
\begin{aligned}
x^{\prime}(t) & =\left\{P(t)^{-1}[A+V(t)+R(t)] P(t)-P(t)^{-1} P^{\prime}(t)\right\} x(t) \\
& =\left[\Lambda(t)+R_{1}(t)\right] x(t) .
\end{aligned}
$$

Consequently, we can apply Levinson's proof [1, pp. 93-94]. Let $e_{k}$ be the unit vector with 1 in the $k$ th position and zero elsewhere. Then there exists a solution $x_{k}(t)$ to (2.2) and a $t_{0} \geqq 0$ so that

$$
\lim _{t \rightarrow \infty} x_{k}(t) \exp \left\{-\int_{t_{0}}^{t} \lambda_{k}(\tau) d \tau\right\}=e_{k}
$$

But $x_{k}(t)=P(t)^{-1} y_{k}(t)$ so that

$$
\lim _{t \rightarrow \infty} y_{k}(t) \exp \left\{-\int_{t_{0}}^{t} \lambda_{k}(\tau) d \tau\right\}=P e_{k}=p_{k},
$$

where $p_{k}$ is the given eigenvector associated with $\lambda_{k}$.

We shall now proceed to the proof of the proposition. Before we do this it will be useful to prove the following lemma.

Lemma. Suppose $A, V(t)$, and $R(t)$ satisfy the hypotheses of the theorem. Then there exists an invertible matrix $S(t)$ so that for all sufficiently large $t$

$$
S(t)^{-1}[A+V(t)] S(t)=\Lambda(t),
$$

$S(t) \rightarrow P$ as $t \rightarrow \infty, S^{\prime}(t)=S_{1}(t)+S_{2}(t)$, where $\left|S_{1}(t)\right|,\left|S_{2}(t)\right|^{2}$, and $\left|S_{2}^{\prime}(t)\right|$ are integrable, and the diagonal elements of $S(t)^{-1} S^{\prime}(t)$ vanish.

Proof. Let

$$
f(\lambda)=\prod_{j=1}^{n}\left(\lambda-\lambda_{j}\right)
$$

be (up to a factor $(-1)^{n}$ ) the characteristic polynomial of $A$, and

$$
f_{k}(\lambda)=\prod_{j=1}^{n} k\left(\lambda-\lambda_{j}\right)
$$


where the superscript $k$ on $\prod$ means we do not allow $j$ to take on the value $k$ in the product. Similarly, let

$$
f(\lambda, t)=\prod_{j=1}^{n}\left(\lambda-\lambda_{j}(t)\right)
$$

be (up to a factor $(-1)^{n}$ ) the characteristic polynomial of $A+V(t)$ and

$$
f_{k}(\lambda, t)=\prod_{j=1}^{n} k\left(\lambda-\lambda_{j}(t)\right)
$$

Let $\left\{p_{k}: 1 \leqq k \leqq n\right\}$ be a given linearly independent set of eigenvectors for $A$ and let $h_{k}=\left[\partial f\left(\lambda_{k}\right) / \partial \lambda\right]^{-1} p_{k}$. Let us set

$$
q_{k}(t)=f_{k}(A+V(t), t) h_{k}=\prod_{j=1}^{n} k\left(A+V(t)-\lambda_{j}(t)\right) h_{k} .
$$

Clearly $q_{k}(t) \rightarrow p_{k}$ as $t \rightarrow \infty$, and

$$
\left(A+V(t)-\lambda_{k}(t)\right) q_{k}(t)=\prod_{j=1}^{n}\left(A+V(t)-\lambda_{j}(t)\right) h_{k}=0 .
$$

Thus $q_{k}(t)$ is an eigenvector associated with $\lambda_{k}(t)$.

Let us set $Q(t)=\left[q_{1}(t), \ldots, q_{k}(t)\right]$, where this means that the $k$ th column of $Q(t)$ is the vector $q_{k}(t)$. For all sufficiently large $t$, the set $\left\{q_{k}(t): 1 \leqq k \leqq n\right\}$ is linearly independent. Hence,

$$
Q(t)^{-1}(A+V(t)) Q(t)=\Lambda(t) .
$$

If we differentiate the last equation with respect to $t$ we get

$$
Q(t)^{-1} V^{\prime}(t) Q(t)=\Lambda^{\prime}(t)-\left[\Lambda(t), Q(t)^{-1} Q^{\prime}(t)\right],
$$

where the last term is the Lie product

$$
\left[\Lambda(t), Q(t)^{-1} Q^{\prime}(t)\right]=\Lambda(t) Q(t)^{-1} Q^{\prime}(t)-Q(t)^{-1} Q^{\prime}(t) \Lambda(t) .
$$

Since $\left[\Lambda(t), Q(t)^{-1} Q^{\prime}(t)\right]$ has zeros down the main diagonal it follows from (2.6) that $\lambda_{j}^{\prime}(t)$ is the $j, j$ entry of $Q(t)^{-1} V^{\prime}(t) Q(t)$. Thus if we write $V^{\prime}(t)=V_{1}^{\prime}(t)+V_{2}^{\prime}(t)$ we see that

$$
\lambda_{j}^{\prime}(t)=\lambda_{1 j}(t)+\lambda_{2 j}(t) \quad \text { where } \lambda_{1 j}(t), \lambda_{2 j}^{2}(t) \text { and } \lambda_{2 j}^{\prime}(t) \in L^{1} .
$$

If we go back to (2.4) and differentiate we get

$$
q_{k}^{\prime}(t)=\sum_{l=1}^{n} k \prod_{j=1}^{l-1} k\left(A+V(t)-\lambda_{j}(t)\right)\left(V^{\prime}(t)-\lambda_{l}^{\prime}(t)\right) \prod_{j=l+1}^{n} k\left(A+V(t)-\lambda_{j}(t)\right) h_{k},
$$

where the superscript $k$ on the summation sign means that $l$ does not take on the 
value $k$. If $l=1$ or $l=n$, the corresponding term is 1 . Let us put

$$
\begin{aligned}
& q_{1 k}(t)=\sum_{l=1}^{n} k \prod_{j=1}^{l-1} k\left(A+V(t)-\lambda_{j}(t)\right)\left(V_{1}^{\prime}(t)-\lambda_{1 l}(t)\right) \prod_{j=l+1}^{n}\left(A+V(t)-\lambda_{j}(t)\right) h_{k}, \\
& q_{2 k}(t)=\sum_{l=1}^{n} k \prod_{j=1}^{l-1} \prod^{k}\left(A+V(t)-\lambda_{j}(t)\right)\left(V_{2}^{\prime}(t)-\lambda_{2 l}(t)\right) \prod_{j=l+1}^{n} \prod^{k}\left(A+V(t)-\lambda_{j}(t)\right) h_{k},
\end{aligned}
$$

and

$$
\begin{aligned}
& Q_{1}(t)=\left[q_{11}(t), q_{12}(t), \ldots, q_{1 n}(t)\right], \\
& Q_{2}(t)=\left[q_{21}(t), q_{22}(t), \ldots, q_{2 n}(t)\right] .
\end{aligned}
$$

Clearly $Q^{\prime}(t)=Q_{1}(t)+Q_{2}(t)$. From (2.7) and the hypotheses of the theorem it follows that $\left|Q_{1}(t)\right|,\left|Q_{2}(t)\right|^{2}$ and $\left|Q_{2}^{\prime}(t)\right|$ are integrable. We shall leave these elementary computations to the reader.

Let us now compute the diagonal elements of $Q(t)^{-1} Q^{\prime}(t)$. From (2.5) we get

$$
Q(t)^{-1}(A+V(t))=\Lambda(t) Q(t)^{-1}
$$

Thus we get

$$
Q(t)^{-1} Q(t)=\left[\prod_{j=1}^{n} 1\left(\Lambda(t)-\lambda_{j}(t)\right) Q(t)^{-1} h_{1}, \ldots, \prod_{j=1}^{n} n\left(\Lambda(t)-\lambda_{j}(t)\right) Q(t)^{-1} h_{n}\right] .
$$

A simple computation shows that

$$
\left(Q(t)^{-1} Q(t)\right)_{k, k}=\left(\partial f\left(\lambda_{k}(t), t\right) / \partial \lambda\right)\left(Q(t)^{-1} h_{k}\right)_{k}=1,
$$

where $\left(Q(t)^{-1} h_{k}\right)_{k}$ is the $k$ th component of the vector $Q(t)^{-1} h_{k}$. Since

we get

$$
\partial f\left(\lambda_{k}(t), t\right) / \partial \lambda \neq 0
$$

Using (2.5) and (2.9), the $k$ th column of the matrix $Q(t)^{-1} Q^{\prime}(t)$ is given by

$$
\begin{aligned}
\left(Q(t)^{-1} Q^{\prime}(t)\right)_{k}= & \sum_{l=1}^{n} \prod_{j=1}^{l-1} \prod^{k}\left(\Lambda(t)-\lambda_{j}(t)\right)\left(Q(t)^{-1} V^{\prime}(t) Q(t)-\lambda_{l}^{\prime}(t)\right) \\
& \times \prod_{j=l+1}^{n}\left(\Lambda(t)-\lambda_{j}(t)\right) Q(t)^{-1} h_{k} .
\end{aligned}
$$

We want to compute the $k$ th component of this vector. To do this we first use (2.6) in (2.11) to obtain

$$
\begin{aligned}
\left(Q(t)^{-1} Q^{\prime}(t)\right)_{k} \\
=\sum_{l=1}^{n} k \prod_{j=1}^{l-1} k\left(\Lambda(t)-\lambda_{j}(t)\right)\left(\Lambda^{\prime}(t)-\lambda_{l}^{\prime}(t)\right) \prod_{j=l+1}^{n}\left(\Lambda(t)-\lambda_{j}(t)\right) Q(t)^{-1} h_{k} \\
\quad-\sum_{l=1}^{n} \prod_{j=1}^{l-1} k\left(\Lambda(t)-\lambda_{j}(t)\right)\left(\left[\Lambda(t), Q(t)^{-1} Q^{\prime}(t)\right]\right) \\
\quad \times \prod_{j=l+1}^{n}\left(\Lambda(t)-\lambda_{j}(t)\right) Q(t)^{-1} h_{k} .
\end{aligned}
$$


The $k$ th component of the first summation is easily computed. Indeed, using (2.10) the $k$ th component is given by

$$
\begin{aligned}
\sum_{l=1}^{n} k\left(\lambda_{k}^{\prime}(t)-\lambda_{l}^{\prime}(t)\right) & \prod_{j=1}^{n} k, l\left(\lambda_{k}(t)-\lambda_{j}(t)\right)\left(Q(t)^{-1} h_{k}\right)_{k} \\
& =\frac{d}{d t}\left(\frac{\partial f\left(\lambda_{k}(t), t\right)}{\partial \lambda}\right) / \frac{\partial f\left(\lambda_{k}(t), t\right)}{\partial \lambda}=\frac{d}{d t}\left(\ln \frac{\partial f\left(\lambda_{k}(t), t\right)}{\partial \lambda}\right),
\end{aligned}
$$

where the superscript $k, l$ on the product sign means that $j$ does not take on the values $k$ and $l$.

Let $\mu_{k}(t)$ be the $k$ th component of the second vector sum. We claim that $\mu_{k}(t)$ is integrable. To see this let us look at the lth term in this sum. Since

$$
\left[\Lambda(t), Q(t)^{-1} Q^{\prime}(t)\right]
$$

has zeros down its main diagonal and since $\Lambda(t)-\lambda_{j}(t)$ is a diagonal matrix, the $k$ th row of

$$
\prod_{j=1}^{l-1} k\left(\Lambda(t)-\lambda_{j}(t)\right)\left(\left[\Lambda(t), Q(t)^{-1} Q^{\prime}(t)\right]\right) \prod_{j=l+1}^{n} k\left(\Lambda(t)-\lambda_{j}(t)\right)
$$

has a zero in the $k$ th position. Since $P^{-1} h_{k}$ has zero in every entry except the $k$ th position it follows that the $k$ th component of the $l$ th term in the second sum of (2.12) is the same as the $k$ th component of the vector

$$
\prod_{j=1}^{l-1} k\left(\Lambda(t)-\lambda_{j}(t)\right)\left(\left[\Lambda(t), Q(t)^{-1} Q^{\prime}(t)\right]\right) \prod_{j=l+1}^{n} k\left(\Lambda(t)-\lambda_{j}(t)\right)\left(Q(t)^{-1}-P^{-1}\right) h_{k} .
$$

Now, we claim that $\left|Q(t)^{-1}-P^{-1}\right|=O(|V(t)|)$. To show this it will be enough to show that

$$
|Q(t)-P|=O(|V(t)|)
$$

From the definition of $Q(t)$ and $P$ we have

$$
q_{k}(t)-p_{k}=\prod_{j=1}^{n} k\left(A+V(t)-\lambda_{j}(t)\right) h_{k}-\prod_{j=1}^{n} k\left(A-\lambda_{j}\right) h_{k} .
$$

Since $A h_{k}=\lambda_{k} h_{k}$ we have

$$
\begin{aligned}
\prod_{j=1}^{n} k\left(A+V(t)-\lambda_{j}(t)\right) h_{k}= & \prod_{j=1}^{n-1} k\left(A+V(t)-\lambda_{j}(t)\right)\left(V(t)+\lambda_{k}-\lambda_{n}(t)\right) h_{k} \\
= & \prod_{j=1}^{n-1} k\left(A+V(t)-\lambda_{j}(t)\right) V(t) h_{k} \\
& +\prod_{j=1}^{n-2} k\left(A+V(t)-\lambda_{j}(t)\right)\left(V(t)+\lambda_{k}-\lambda_{n-1}(t)\right)\left(\lambda_{k}-\lambda_{n}(t)\right) h_{k} .
\end{aligned}
$$


Proceeding by induction we see that

$$
\prod_{j=1}^{n} k\left(A+V(t)-\lambda_{j}(t)\right) h_{k}=M(t) V(t) h_{k}+\prod_{j=1}^{n} k\left(\lambda_{k}-\lambda_{j}(t)\right) h_{k},
$$

where $|M(t)|$ is uniformly bounded. Thus, from (2.15) we get

$$
q_{k}(t)-p_{k}=M(t) V(t) h_{k}+\left\{\prod_{j=1}^{n} k\left(\lambda_{k}-\lambda_{j}(t)\right)-\prod_{j=1}^{n} k\left(\lambda_{k}-\lambda_{j}\right)\right\} h_{k} .
$$

We may write

$$
\prod_{j=1}^{n} k\left(\lambda_{k}-\lambda_{j}(t)\right)-\prod_{j=1}^{n} k\left(\lambda_{k}-\lambda_{j}\right)=\sum_{j=1}^{n} g_{j}(t)\left(\lambda_{j}-\lambda_{j}(t)\right),
$$

where each $g_{j}(t)$ is a bounded numerical valued function.

We claim that

$$
\lambda_{j}-\lambda_{j}(t)=O(|V(t)|) .
$$

To see this let us write $f(\lambda, t)=\sum_{k=0}^{n} \kappa_{k}(t) \lambda^{k}$ where the $\kappa_{k}(t)$ are analytic functions of the entries of $A+V(t)$. If $\kappa_{k}$ are the coefficients of $f(\lambda)$, then of course

$$
f\left(\lambda_{j}\right)=\sum_{k=0}^{n} \kappa_{k} \lambda_{j}^{k}=0, \quad 1 \leqq j \leqq n .
$$

Also, since the roots of $f(\lambda)$ are simple, $\partial f\left(\lambda_{j}\right) / \partial \lambda \neq 0,1 \leqq j \leqq n$. Now let us consider the function of $n+2$ complex variables $F\left(\lambda, c_{0}, \ldots, c_{n}\right)=\sum_{k=0}^{n} c_{k} \lambda^{k}$. We have that

$$
\begin{aligned}
F\left(\lambda_{j}, \kappa_{0}, \ldots, \kappa_{n}\right) & =f\left(\lambda_{j}\right)=0, \\
\partial F\left(\lambda_{j}, \kappa_{0}, \ldots, \kappa_{n}\right) / \partial \lambda & =\partial f\left(\lambda_{j}\right) / \partial \lambda \neq 0 .
\end{aligned}
$$

Since $F$ is an analytic function we may use the implicit function theorem to "solve" the equation $F\left(\lambda, c_{0}, \ldots, c_{n}\right)=0$ for $\lambda$ as an analytic function of $\left(c_{0}, \ldots, c_{n}\right)$ in some polydisc about the point $\left(\kappa_{0}, \ldots, \kappa_{n}\right)$ so that $\lambda\left(\kappa_{0}, \ldots, \kappa_{n}\right)=\lambda_{j}$. Let us designate this function by $\lambda_{j}\left(c_{0}, \ldots, c_{n}\right)$.

Since $\kappa_{k}(t) \rightarrow \kappa_{k}$ and $\lambda_{j}(t) \rightarrow \lambda_{j}$ as $t \rightarrow \infty$, we see that, for all sufficiently large $t$, $F\left(\lambda_{j}(t), \kappa_{0}(t), \ldots, \kappa_{n}(t)\right)=0$; i.e., $\lambda_{j}(t)=\lambda_{j}\left(\kappa_{0}(t), \ldots, \kappa_{n}(t)\right)$. Thus, for all sufficiently large $t$ we may write

$$
\lambda_{j}(t)-\lambda_{j}=\sum_{k=0}^{n}\left(\kappa_{k}(t)-\kappa_{k}\right) w_{k}(t),
$$

where $w_{k}(t)$ is a bounded function. Further,

$$
\kappa_{k}(t)-\kappa_{k}=\sum_{s=1}^{n} \sum_{r=1}^{n} v_{r, s}(t) u_{r, s}(t)
$$

where the $v_{r, s}(t)$ are the entries of $V(t)$ and the $u_{r, s}(t)$ are bounded functions. If we use this in (2.19) we see that (2.18) is satisfied. If we use this fact in (2.17), it follows 
from (2.16) that (2.14) is satisfied. If we use this in (2.13), we see that the $k$ th component $\mu_{k}(t)$ of the second sum of $(2.12)$ is

$$
\mu_{k}(t)=O\left(\left|Q^{\prime}(t)\right||V(t)|\right) .
$$

From (2.8) and $\left(2.8^{\prime}\right)$ we may write $Q^{\prime}(t)=Q_{1}(t)+Q_{2}(t)$ where $\left|Q_{1}(t)\right|=O\left(\left|V_{1}^{\prime}(t)\right|\right)$ and $\left|Q_{2}(t)\right|=O\left(\left|V_{2}^{\prime}(t)\right|\right)$. If we use these facts in (2.20), and the hypotheses of the theorem, we see that $\mu_{k}(t)$ is integrable.

To finish the proof of the lemma we must now construct the matrix valued function $S(t)$. We have, up to this point, constructed a matrix valued function $Q(t)$ which has all of the properties of the function $S(t)$ of the lemma with the possible exception that the diagonal elements of $Q(t)^{-1} Q^{\prime}(t)$ are not zero. Indeed we have

$$
\begin{aligned}
\left(Q(t)^{-1} Q^{\prime}(t)\right)_{k, k} & =\frac{d}{d t}\left(\ln \frac{\partial f\left(\lambda_{k}(t), t\right)}{\partial \lambda}\right)+\mu_{k}(t) \\
& =\frac{d}{d t}\left(\ln \left\{\frac{\partial f\left(\lambda_{k}(t), t\right)}{\partial \lambda} \exp \left[\int_{0}^{t} \mu_{k}(\tau) d \tau-\int_{0}^{\infty} \mu_{k}(\tau) d \tau\right]\right\}\right) \\
& =\frac{d}{d t}\left(\ln G_{k}(t)\right),
\end{aligned}
$$

where it is clear how $G_{k}(t)$ is defined.

Let us set

$$
s_{k}(t)=\left(\partial f\left(\lambda_{k}\right) / \partial \lambda\right) G_{k}(t)^{-1} q_{k}(t) .
$$

It is clear that $s_{k}(t)$ is an eigenvector for $A+V(t)$ associated with $\lambda_{k}(t)$ and, for all sufficiently large $t$, the set of vectors $\left\{s_{k}(t): 1 \leqq k \leqq n\right\}$ is linearly independent. Hence, for all sufficiently large $t, S(t)$ may be used to diagonalize $A+V(t)$. Further, it is clear that $s_{k}(t) \rightarrow p_{k}$ as $t \rightarrow \infty$.

If we differentiate $s_{k}(t)$ we get

$$
s_{k}^{\prime}(t)=\frac{\partial f\left(\lambda_{k}\right)}{\partial \lambda} \frac{d G_{k}(t)^{-1}}{d t} q_{k}(t)+\frac{\partial f\left(\lambda_{k}\right)}{\partial \lambda} G_{k}(t)^{-1} q_{k}^{\prime}(t) .
$$

Consequently,

$$
S(t)^{-1} s_{k}^{\prime}(t)=\frac{\partial f\left(\lambda_{k}\right)}{\partial \lambda} \frac{d G_{k}(t)^{-1}}{d t} S(t)^{-1} q_{k}(t)+\frac{\partial f\left(\lambda_{k}\right)}{\partial \lambda} G_{k}(t)^{-1} S(t)^{-1} q_{k}^{\prime}(t)
$$

The $k$ th row of $S(t)^{-1}$ consists of the cofactors of the $k$ th column of $S(t)$ divided by $\operatorname{det} S(t)$. Further,

$$
\operatorname{det} S(t)=\prod_{k=1}^{n} \frac{\partial f\left(\lambda_{k}\right)}{\partial \lambda} G_{k}(t)^{-1} \operatorname{det} Q(t)
$$

and the cofactor $\operatorname{Co}\left(s_{k}(t)\right)_{j}$ is given by

$$
\operatorname{Co}\left(s_{k}(t)\right)_{j}=\left\{\prod_{l=1}^{n} \frac{\partial f\left(\lambda_{l}\right)}{\partial \lambda} G_{l}(t)^{-1}\right\} \operatorname{Co}\left(q_{k}(t)\right)_{j}
$$


Hence, we get

$$
S(t)^{-1} q_{k}(t)=\left\{\partial f\left(\lambda_{k}\right) / \partial \lambda\right\}^{-1} G_{k}(t) Q(t)^{-1} q_{k}(t) .
$$

Thus the first term in (2.24) is $-(d / d t) \ln G_{k}(t) e_{k}$, where $e_{k}$ is the unit vector with 1 in the $k$ th position and zero elsewhere. Using the same argument the second term in (2.24) is $Q(t)^{-1} q_{k}^{\prime}(t)$. Hence, the $k$ th component of the vector (2.22) is

$$
\left(S(t)^{-1} S^{\prime}(t)\right)_{k, k}=-(d / d t) \ln G_{k}(t)+\left(Q(t)^{-1} Q^{\prime}(t)\right)_{k, k} \text {. }
$$

If we add both sides of (2.21) and (2.25) we see that $\left(S(t)^{-1} S^{\prime}(t)\right)_{k, k}=0$.

To finish the proof of the lemma it remains to establish the integrability conditions on $S^{\prime}(t)$. If we refer to (2.23) we first note that $q_{k}^{\prime}(t)$ breaks up in the required way. Consequently, if the derivative of $G_{k}(t)$ breaks up in a suitable way, then so does $s_{k}^{\prime}(t)$. However, an easy computation shows that if we use the hypothesis on $V^{\prime}(t),(2.7)$, and the definition of $\mu_{k}(t)$ via the second sum in (2.12), this is indeed the case. Hence, the proof of the lemma is complete.

Proof of the Proposition. Let $S(t)$ be the matrix of the previous lemma. Make the transformation $y(t)=S(t) z(t)$. Then from (1.1) we get

$$
\begin{aligned}
z^{\prime}(t) & =\left[S(t)^{-1}\{A+V(t)\} S(t)-S(t)^{-1} S^{\prime}(t)+S(t)^{-1} R(t) S(t)\right] z(t) \\
& =\left[\Lambda(t)-S(t)^{-1} S_{2}(t)+R_{0}(t)\right] z(t),
\end{aligned}
$$

where $R_{0}(t)$ is integrable.

We now make use of Fedorjuk's idea. Let $T(t)$ be an $n \times n$ matrix valued function, absolutely continuous on every compact subinterval of $(0, \infty)$ so that $|T(t)|<1$. Make the transformation $z(t)=[I+T(t)] x(t)$, and since $|T(t)|<1,[I+T(t)]^{-1}$ exists and from (2.26) we get

$$
x^{\prime}(t)=[I+T(t)]^{-1}\left[\Lambda(t)-S(t)^{-1} S_{2}(t)-T^{\prime}(t)[I+T(t)]^{-1}+R_{0}(t)\right][I+T(t)] x(t) .
$$

We may write

$$
[I+T(t)]^{-1}=I-T(t)+T^{2}(t)[I+T(t)]^{-1} .
$$

Using this in the equation for $x^{\prime}(t)$ we get

$$
x^{\prime}(t)=\left\{\Lambda(t)+[\Lambda(t), T(t)]-S(t)^{-1} S_{2}(t)+R_{2}(t)\right\} x(t),
$$

where $[\Lambda(t), T(t)]$ is the Lie product and

$$
\begin{aligned}
R_{2}(t)= & -T(t) \Lambda(t) T(t)+T^{2}(t)[I+T(t)]^{-1} \Lambda(t)[I+T(t)] \\
& +T(t) S(t)^{-1} S_{2}(t) T(t)+\left[T(t), S(t)^{-1} S_{2}(t)\right] \\
& +T^{2}(t)[I+T]^{-1} S(t)^{-1} S_{2}(t)[I+T] \\
& +[I+T(t)]^{-1}\left[R_{0}(t)-T^{\prime}(t)[I+T(t)]^{-1}\right][I+T(t)] .
\end{aligned}
$$

Now, $[\Lambda(t), T(t)]$ has zeros down the main diagonal and its $j, k$ component is of the form

$$
[\Lambda(t), T(t)]_{j, k}=\left(\lambda_{j}(t)-\lambda_{k}(t)\right) T(t)_{j, k} .
$$


If we set

$$
T(t)_{j, k}=\left(\lambda_{j}(t)-\lambda_{k}(t)\right)^{-1}\left(S(t)^{-1} S_{2}(t)\right)_{j, k}, \quad T(t)_{j, j}=0,
$$

then

$$
\Lambda_{1}(t)=[\Lambda(t), T(t)]-S(t)^{-1} S_{2}(t)
$$

is diagonal with entries $-\left(S(t)^{-1} S_{2}(t)\right)_{j, j}$. Since

$$
|T(t)| \leqq \max _{j, k}\left|\lambda_{j}(t)-\lambda_{k}(t)\right|^{-1}\left|S(t)^{-1}\right|\left|S_{2}(t)\right|,
$$

we see $\left({ }^{3}\right)$ that $T(t) \rightarrow 0$ as $t \rightarrow \infty$; indeed

$$
|T(t)|=O\left(\left|S_{2}(t)\right|\right) .
$$

Furthermore, if $j \neq k$,

$$
\begin{aligned}
T^{\prime}(t)_{j, k}= & \left(-S(t)^{-1} S^{\prime}(t) S(t)^{-1} S_{2}(t)+S(t)^{-1} S_{2}^{\prime}(t)\right)_{j, k} /\left(\lambda_{j}(t)-\lambda_{k}(t)\right) \\
& -\left(\lambda_{j}^{\prime}(t)-\lambda_{k}^{\prime}(t)\right)\left(S(t)^{-1} S_{2}(t)\right)_{j, k} /\left(\lambda_{j}(t)-\lambda_{k}(t)\right)^{2} .
\end{aligned}
$$

Since $S^{\prime}(t)=S_{1}(t)+S_{2}(t)$, we get

$$
\left|S(t)^{-1} S^{\prime}(t) S(t)^{-1} S_{2}(t)\right|=O\left(\left|S_{1}(t)\right|+\left|S_{2}(t)\right|^{2}\right) .
$$

Also,

$$
\begin{aligned}
\left|\lambda_{j}^{\prime}(t)-\lambda_{k}^{\prime}(t)\right| & \left|\left(S(t)^{-1} S_{2}(t)\right)_{j, k}\right| \\
& \leqq\left\{\left|\lambda_{1 j}(t)-\lambda_{1 k}(t)\right|+\left|\lambda_{2 j}(t)-\lambda_{2 k}(t)\right|\right\}\left|S(t)^{-1}\right|\left|S_{2}(t)\right| .
\end{aligned}
$$

From (2.7) and the fact that $\left|S_{2}(t)\right| \in L^{2}$, we see that the left-hand side of the above inequality is integrable. Using all of this information in (2.31) we see that

$$
\left|T^{\prime}(t)\right| \in L^{1} \text {. }
$$

Going back to (2.28), using (2.30) and the fact that $\left|S_{2}(t)\right| \in L^{2}$, and using (2.32) we see that $\left|R_{2}(t)\right| \in L^{1}$.

It is necessary to compute the diagonal elements of $S(t)^{-1} S_{2}(t)$. However, it is enough to compute the diagonal elements of $S(t)^{-1} S^{\prime}(t)$, since these differ from the diagonal elements of $S(t)^{-1} S_{2}(t)$ by integrable functions, namely the diagonal elements of $S(t)^{-1} S_{1}(t)$, which we can add to $R_{2}(t)$ to get an integrable matrix valued function $R_{1}(t)$. From the previous lemma, $\left(S(t)^{-1} S^{\prime}(t)\right)_{k, k}=0$.

If we now set $P(t)=S(t)[I+T(t)]$ we have completed the proof of the proposition.

Finally, at the referee's suggestion (a good one), we shall give a simple example to show that the class of matrices $V(t)$ given in the hypothesis of our theorem includes, as a proper subclass, the class of $V(t)$ given in the hypothesis of Levinson's theorem. We shall give an example of a numerical valued function since it is clear

$\left(^{3}\right)$ We note at this point that in view of the hypotheses on $V_{2}^{\prime}, V_{2}^{\prime}(t) \rightarrow 0$ as $t \rightarrow \infty$, and hence the same is true for $S_{2}$. 
that matrices satisfying our hypotheses, but not Levinson's, can be constructed from such numerical valued functions. Let us take

$$
V(t)=-\int_{t^{\alpha}}^{\infty} \frac{\sin \tau}{\tau} d \tau, \quad \frac{1}{2}<\alpha<1 .
$$

Clearly, $V(t) \rightarrow 0$ as $t \rightarrow \infty$. If we integrate by parts twice we get

$$
V(t)=-\left(\cos t^{\alpha}\right) / t^{\alpha}+O\left(1 / t^{2 \alpha}\right) .
$$

This shows, first of all, that $V(t) \notin L^{1}(1, \infty)$. Next we have

$$
\begin{aligned}
V^{\prime}(t)= & \left(\alpha \sin t^{\alpha}\right) / t \notin L^{1}(1, \infty) ; \quad\left|V^{\prime}\right|^{2} \in L^{1}(1, \infty) ; \\
V^{\prime \prime}(t)= & \left(\alpha^{2} \cos t^{\alpha}\right) / t^{2-\alpha}-\left(\alpha \sin t^{\alpha}\right) / t^{2} \in L^{1}(1, \infty) ; \\
& V^{\prime}(t) V(t)=O\left(1 / t^{1+\alpha}\right) \in L^{1}(1, \infty) .
\end{aligned}
$$

\section{REFERENCES}

1. E. A. Coddington and N. Levinson, Theory of ordinary differential equations, McGrawHill, New York, 1955. MR 16, 1022.

2. A. Devinatz, The deficiency index of a certain class of ordinary self-adjoint differential operators, Advances in Math. (to appear).

3. - The deficiency index of ordinary self-adjoint differential operators, Pacific J. Math. 16 (1966), 243-257. MR 32 \#2688.

4. M. V. Fedorjuk, Asymptotic methods in the theory of one-dimensional singular differential operators, Trudy Moskov. Mat. Obšč. 15 (1966), 296-345 = Trans. Moscow Math. Soc. 1966, 333-386. MR 34 \#7870.

5. N. Levinson, The asymptotic nature of solutions of linear systems of differential equations, Duke Math. J. 15 (1948), 111-126. MR 9, 509.

NORTHWESTERN UNIVERSITY, EVANSTON, ILLINOIS 60201 